\title{
La identidad epistemológica de los estudiantes de doctorado en Educación
}

\author{
The epistemological identity of students of Education Doctorate
}

\author{
Adrián Hernández Vélez
}

\begin{abstract}
RESUMEN
En este texto se analizan los factores que conforman la identidad epistemológica de estudiantes del doctorado en Educación de una institución privada en la ciudad de Acapulco, Guerrero (México). El posicionamiento teórico se construyó reconociendo a la identidad como un objeto de estudio multifacético, posible de abordar desde diferentes disciplinas; en cuanto a la epistemología, se recuperan las principales escuelas que fundamentan la génesis del conocimiento. El posicionamiento epistémico del estudio es de tipo fenomenológico y la arquitectura metodológica fue de tipo cualitativo, con un diseño hermenéutico, utilizando como técnica la entrevista. Entre los principales hallazgos es posible reconocer que, para la conformación de su identidad epistemológica, los investigadores del campo educativo requieren comprender el estado de desarrollo de su propia identidad docente, así como el reconocimiento de la no-neutralidad ideológica en el quehacer investigativo.
\end{abstract}

Palabras clave: educación, epistemología, identidad, investigación.

\begin{abstract}
In this text we analyze the factors that make up the epistemological identity of Doctoral students in Education of a private institution in the city of Acapulco, Guerrero (Mexico). The theoretical positioning was constructed recognizing identity as an object of multifaceted study, possible to approach from different disciplines; as for epistemology, the main schools that support the genesis of knowledge are recovered. The epistemological positioning of the study is phenomenological, the methodological architecture was qualitative, with a hermeneutical design using the interview as a technique. Among the main findings it is possible to recognize that, for the conformation of an epistemological identity, researchers in the educational field need to understand the state of development of their own teaching identity, as well as the recognition of ideological non-neutrality in the research work.
\end{abstract}

Keywords: education, epistemology, identity, research. 


\section{INTRODUCCIÓN}

El doctorado es el máximo grado académico que una institución de educación superior otorga a quienes cursan programas de posgrado. En el contexto internacional los programas de doctorado presentan diferentes niveles de consolidación debido al estado de desarrollo del campo del conocimiento que indagan, así como por el impacto del marco político que regula su función en cada contexto (Núñez-Valdés y González, 2019). En el caso mexicano los estudios de posgrado han experimentado cambios trascendentales en las últimas tres décadas, entre estos la expansión y diversificación de su oferta, así como su regulación con base en políticas de evaluación con énfasis cuantitativo, a través de las cuales se ha buscado fortalecer su calidad (García, 2009). Aun así, también se agudizaron diferencias institucionales, tales como la falta de equilibrio en la distribución de la matrícula por área de conocimiento, así como su poca vinculación con sectores sociales y productivos (Álvarez, 2002).

Los programas de doctorado con énfasis en investigación, aunque cuentan con un marco político articulador que promueve su calidad, presentan distintos niveles de desarrollo, así como cambios que dependen de su naturaleza disciplinar e institucional. Su reconocimiento lo otorga la Secretaría de Educación Pública (SEP) y en particular tienen la misión de formar investigadores que obtienen el grado acreditando los cursos que forman la base curricular de su programa de adscripción y desarrollando una investigación rigurosa a través de la cual generan nuevos conocimientos o construyen nuevos sentidos al conocimiento existente.

Para Möller y Gómez (2014), estos programas han formado doctores con alto rigor académico sobre una lógica de resultados, conforme a la cual la habilitación de posgraduados constituye un valor social que fortalece la competitividad de las naciones bajo el argumento de que a mayor generación de conocimientos corresponde un mayor desarrollo económico (Fernández y Wainerman, 2015). Es un enfoque que, desde la década de 1990, con base en las políticas del Estado evaluador, promovió un modelo para la mejora y consolidación de la calidad de las instituciones y programas educativos, soportado en la planeación estratégica, evaluación y rendición de cuentas (Neave, 2001; Mendoza, 2002).

Sin embargo, para comprender la trascendencia de este tipo de doctorados, además de considerar la lógica de resultados, se requiere tomar en cuenta los factores que

Adrián Hernández Vélez. Profesor en el doctorado en educación en el Instituto de Estudios Universitarios (IEU), sede Puebla, México. Es doctor en Educación por la Universidad Autónoma de Tlaxcala (UATx) y colabora en los posgrados en Educación de la Universidad de las Américas Puebla (UDLAP). Entre sus publicaciones más recientes se encuentra el libro Repensar la educación en México. Textos a deliberación (coordinador, 2019) y el artículo "Los consejos técnicos escolares en Morelos. Expectativas y tensiones en la zona 40”, publicado en la revista Praxis Educativa de la REDIE (2019). Correo electrónico: ahv31@hotmail. com. ID: https://orcid.org/0000-0003-0940-010X. 
intervienen en los procesos formativos que experimentan los estudiantes en su trayectoria escolar, por ejemplo, el estado de desarrollo de cada campo del conocimiento, la relación entre el campo profesional y el objeto de estudio elegido, las tradiciones metodológicas que prevalecen en cada programa, así como la revisión del nivel de cumplimiento en la construcción del perfil de egreso (Fresán, 2002). Conforme a este último, resulta preciso considerar que la valoración sobre la calidad con la que son formados los estudiantes no puede quedar acotada a la revisión final del nivel de cumplimiento de su perfil de egreso, por el contrario, requiere comprender asuntos medulares relacionados a su habilitación doctoral, por eso, tratándose de un grado y modalidad que forma investigadores, en principio debe indagarse cómo posicionan sus intenciones de generación de nuevos conocimientos, lo cual se encuentra en el campo de la epistemología (Gadamer, 2001; Osorio, Páramo, Otálvaro, Posada, Henríquez, Barriga, Hernández, Romero, Bracho, Raymond, Cárcamo, Gavarotto, Arnold, Pájaro-Huertas, Martínez, Scríbano y Toledo, 2007).

La epistemología constituye el corpus encargado de señalar qué es el conocimiento y cómo se genera, por ende, permite precisar cuáles son las intenciones y rutas emprendidas para su desarrollo. Si un estudiante de doctorado se forma como investigador, primero debe argumentar en qué escuelas epistemológicas se posiciona frente a la realidad, lo cual orientará todo proceso de corte metodológico y la construcción de sentido a los datos, pudiendo afirmar por qué sus hallazgos pueden ser considerados como conocimientos (Lora y Sánchez, 2012).

En esta investigación se indagó el proceso formativo, conforme al cual se buscó conocer quiénes son los estudiantes del doctorado en educación del Instituto de Estudios Universitarios (IEU) campus Acapulco, así como los factores que intervienen en la conformación de su identidad epistemológica. Se partió de la tesis de que los doctorados en educación que tienen un enfoque de investigación forman investigadores que asumen como misión la generación de nuevos conocimientos o la construcción de nuevos sentidos al conocimiento existente, lo cual requiere que, en su carácter de investigadores en formación, precisen cuáles son las bases epistemológicas desde las cuales se posicionan.

En cuanto al contexto, el IEU es una organización educativa privada, fundada en 1976, cuya misión radica en formar profesionistas de pregrado y posgrado con conocimientos, habilidades, valores y actitudes que les permitan construirse a sí mismos y contribuir al desarrollo social. Su oferta abarca programas de licenciatura, maestría y doctorado, impartidos en las modalidades presencial, ejecutiva y a distancia. Se trata de una escuela certificada por la Federación de Instituciones Mexicanas Particulares de Educación Superior (FIMPES), reconocida por su organización multicampi y tiene como sede principal al campus de la ciudad de Puebla. 
Conforme a la Asociación Nacional de Universidades e Instituciones de Educación Superior (ANUIES), en el estado de Guerrero, en el ciclo escolar 2017-2018, se registró una matrícula de 2,301 estudiantes inscritos en programas de especialidad, maestría y doctorado de instituciones públicas y privadas. En cuanto a los programas de doctorado, la matrícula fue de 236 alumnos, siendo el campo de la educación el que presentó mayor demanda con 148 matriculados en siete programas que abarcan diferentes modalidades curriculares. El doctorado en educación del IEU concentró a 21 de esos estudiantes en su modalidad ejecutiva, la cual articula sesiones presenciales y la asistencia curricular a través de plataformas digitales.

\section{Posicionamiento Teórico}

La ruta teórica contó con dos ejes de análisis que en conjunto permitieron establecer las bases para indagar la identidad epistemológica de los doctorantes en educación: identidad y epistemología. La identidad es un objeto de estudio multifacético, por lo que alrededor de esta ocurre una disputa que abarca diferentes disciplinas, generando en un primer momento la idea de un campo desarticulado; sin embargo no existe tal desarticulación, sino la deconstrucción de su objeto de estudio desde diferentes campos (antropología, sociología, economía, pedagogía, etc.) que dialogan entre sí, logrando establecer puentes teóricos y empíricos que configuran una ruta de interpretación multidisciplinaria.

En este trabajo fue recuperada esa diversidad discursiva, particularmente las aportaciones antropológicas y sociológicas que reconocen a la identidad como una construcción personal y colectiva que está orientada a precisar quién es el sujeto en sí mismo y en su relación con los demás. Para Campos-Winter (2018), en el primer caso se muestra como esencia y proceso. En tanto esencia, consiste en el conjunto de rasgos articulados de un individuo o grupo, así como en el conjunto de símbolos y valores que lo colocan como parte de una comunidad (Torres y Carrasco, 2008). Para Giménez (2010) consiste en aquello que permite establecer fronteras con los otros a partir del reconocimiento del contexto del cual proviene.

La identidad es el reconocimiento de sí mismo en relación con los demás; los grupos están ahí, compuestos por actores que se reconocen como diferentes entre sí, pero que se asumen como parte de un todo que está cohesionado por estructuras de diferente naturaleza (lenguaje, religión, ideología, etc.). Así, individuo y grupo demandan distintas formas de comportamiento sostenidas por una base histórica que los ha dotado de herencias culturales y de un sistema de lenguaje que les permite disputar su propia transformación. El lenguaje aparece como un referente que lleva a los sujetos y a los grupos por el sendero de la comprensión, interpretación y negociación de la realidad y de los símbolos que la definen (Gadamer, 2007). 
En cuanto proceso, consiste en la diferenciación del carácter intersubjetivo, nunca finalizado y siempre cambiante, mediado interactiva y comunicativamente, que permite el reconocimiento de sí mismo y de su autonomía (Vergara, Vergara y Gundermann, 2010). Además se construye desde las interpretaciones de la tradición y desde una relación crítica con esta. Así, la noción de identidad no solo corresponde al reconocimiento de sí mismo sino también a la oposición respecto al otro.

En general, constituye un proceso abierto y dinámico, se trata de un ente inacabado en el cual pueden manifestarse múltiples influencias, no obstante, todas permiten asumirse como parte de una comunidad. Lo crucial radica en saberse parte de esa comunidad y en comprender los factores que perfilan su idiosincrasia dentro de esta y también los que permiten dotarla de cohesión. Zizek (2003) propone una definición de identidad que reúne ambas formas de percibirla. Para él constituye un ente abierto formado por significantes flotantes, cohesionados por un punto nodal; se trata de elementos sueltos en un espacio ideológico abierto, por lo que su significado depende de la capacidad articuladora del punto nodal que los cohesiona dotándolos de sentido.

En cuanto a la epistemología, para Lora y Sánchez (2012) consiste en

[...] la naturaleza del conocimiento en sus principios y funcionamiento, los tipos y los métodos, los procesos cognitivos desde la experiencia, la estructura de la experiencia global de la realidad y el conocimiento coherente, sus manifestaciones en la cultura y la historia [p. 17].

Es la posibilidad de reflexionar desde múltiples ángulos qué es lo que asumimos como conocimiento, es decir, preguntarnos qué significa conocer, cómo conocemos, desde qué ángulo del pensamiento lo hacemos (Llano, 1984), incluso los contextos desde los cuales nos colocamos con la intención de conocer; el hecho de recuperar la primera de esas preguntas puede generar respuestas en diversas vertientes, pero en este caso se retoma la que permite diferenciar entre el conocimiento ordinario y el científico: la epistemología se encarga del estudio del tipo científico, el que se considera como ciencia (Briones, 2002).

Aunque el carácter científico procede de la Grecia antigua, encontró en el Discurso del método de Descartes la guía para definir los procedimientos rigurosos que lo legitiman. El método científico, como derivado de este, tiene que ver con los procedimientos teóricos y observacionales utilizados para generar conocimiento (Castro, Castro y Morales, 2016). Así, desde inicios del siglo XX esta relación epistémico-metodológica encontró en el Positivismo de Comte la base del que hasta la fecha es considerado como el conocimiento legítimo. Aun así, es necesario preguntarnos si acaso la ruta positivista es la única que existe para conocer. Para Gadamer (2007) la verdad no es exclusiva del método cartesiano, por ende, existen otras escuelas que plantean sus propias formas de explicar la génesis del conocimiento. En la tabla 1 se sintetizan las propuestas centrales de las principales escuelas próximas y distantes al Positivismo. 
Tabla 1. Escuelas epistemológicas.

\begin{tabular}{ll}
\hline \multicolumn{1}{c}{ Paradigma } & \multicolumn{1}{c}{ El conocimiento } \\
\hline Materialismo & $\begin{array}{l}\text { Plantea que todo cuanto existe es materia, por lo que, en última instancia, solo existe la realidad material. } \\
\text { La materia es el fundamento de la realidad y de sus transformaciones (Briones, 2002) }\end{array}$ \\
\hline Racionalismo & $\begin{array}{l}\text { Encuentra en el pensamiento, es decir, en la razón, toda fuente de conocimiento humano. El conocimiento } \\
\text { solo será considerado como tal en tanto sea lógicamente necesario y universalmente válido (Verneaux, 1997) }\end{array}$ \\
\hline Idealismo & Consiste en la antítesis del racionalismo y soporta que la única fuente del conocimiento humano se encuentra \\
& en la experiencia, por lo que la mente solo registra el conocimiento que proviene de esta (Hessen, 1982) \\
& $\begin{array}{l}\text { Mantiene una estrecha relación con el racionalismo y sostiene que los objetos físicos no pueden tener } \\
\text { de conocimiento, el idealismo se enfoca sobre su valor (Llano, 1984) }\end{array}$ \\
\hline Fenomenología & $\begin{array}{l}\text { Escuela que sostiene que toda acción de la conciencia está dirigida, y por lo tanto representa una intención, } \\
\text { mediada conforme a lo que se ha planteado para el ser en sí mismo, desempeñando un papel crucial } \\
\text { en la percepción que este tenga sobre el objeto, así la intención recupera la esencia pura de la conciencia } \\
\text { (Lora y Sánchez, 2012) }\end{array}$ \\
\hline $\begin{array}{l}\text { Es una reflexión filosófica universal que se ocupa de la correcta comprensión e interpretación de los textos, } \\
\text { mediada por la historia y el lenguaje. Se manifiesta con desconfianza ante el relativismo que suele tergiversar } \\
\text { el sentido de la verdad. Entre sus principales tesis, sostiene que la verdad no es exclusiva del método científico, } \\
\text { por lo que, en humanidades, existen otras fuentes para generar conocimiento (Gadamer, 2001) }\end{array}$ \\
\hline
\end{tabular}

Fuente: Construcción personal.

Las distintas formas que existen para posicionar la identidad epistemológica se ven condicionadas por las formas en que cada escuela argumenta la naturaleza del conocimiento. Al esclarecerla también se asume la posibilidad de trazar diferencias de corte metodológico, por ejemplo, al conjugar orientaciones epistemológicas y metodológicas fue posible diferenciar las ciencias naturales de las ciencias sociales. Ambas tienen puntos en común, están soportadas en el uso del método científico, la observación es la base de su estructura metodológica y cuentan con rigurosos procedimientos que permiten afirmar que sus resultados conforman conocimiento verdadero. No obstante, su diferencia primordial radica en las intenciones de generación de conocimiento, por eso, aunque las ciencias sociales recurran al método de las ciencias naturales, lo que importa es el sentido del uso que hacen de este.

El campo educativo se ciñe a ambos planteamientos, sin embargo la gran diferencia será, una y otra vez, la base epistemológica que subyace a todo trabajo de corte metodológico; este se ve determinado por los principios de la escuela epistemológica con la cual se identifica el investigador. Por ende, precisar esta identidad implica, como consecuencia, la delimitación de la ruta metodológica, técnica e instrumental desde la cual se aproxima a la realidad. 


\section{Arquitectura metodológica}

El estudio tuvo una base epistémica de tipo fenomenológico y una metodología cualitativa con diseño hermenéutico. ${ }^{1}$ La entrevista fue la técnica a través de la cual se buscó comprender la formación de la identidad epistemológica a partir de la revisión de cuatro ejes: (a) socio-profesional, (b) experiencia en procesos de investigación, (c) posicionamiento epistemológico y (d) idea fuerza del futuro académico.

El posicionamiento epistémico fue de tipo fenomenológico, por lo que el acceso a la realidad estuvo soportado en el concepto de "abstención", lo cual implica el encuentro con los sujetos de investigación en su rol cotidiano, convocando su experiencia para hacerla explícita, develarla al margen de la tradición, lo cual supone despojarse de conceptos o categorías que condicionan su manifestación literal. En lo que corresponde al paradigma de investigación, fue de tipo cualitativo con diseño hermenéutico; se recuperó la hermenéutica de Gadamer (2001), quien asumió que el acceso a la verdad no es exclusivo del método científico, además de situar en el diálogo la posibilidad de encuentro con el otro.

La hermenéutica es una filosofía universal que encuentra en la historia, la cultura y el lenguaje, rutas de acceso a la comprensión e interpretación del mundo (Grondin, 2008). La comprensión e interpretación se construyen en una relación con el otro, a través de instantes en los cuales se encuentran los horizontes y el mensaje se hace posible y se transforma en percepción propia a través del lenguaje (Gadamer, 2000). Este constituyó el medio para la experiencia hermenéutica ya que todo proceso es lingüístico.

El acceso a los datos se dio en dos momentos; el primero correspondió al análisis de la base de información de la institución, conforme a la cual fue posible recuperar los subnúcleos 1.1, 1.2, 1.3 y 1.4. En el segundo momento se desarrolló la reunión con los informantes mediante la técnica de entrevista, la cual consistió en un diálogo regulado por un guión semiestructurado (Kvale, 2011), cuya base se puede observar en la operacionalización de núcleos problemáticos (tabla 2). Fueron invitados todos los estudiantes que se encontraban cursando el cuarto semestre del programa durante el ciclo de otoño 2018, por lo que solo fueron incluidos los que manifestaron su disposición a participar. Se entrevistó a ocho de los once estudiantes matriculados y se les planteó como supuesto inicial la tesis de que su formación doctoral los habilitó para generar nuevos conocimientos.

1 En el campo de la educación suele colocarse a la hermenéutica como una metodología de tipo cualitativo, sin embargo existe un sector que promueve su uso como una epistemología dada su conformación como filosofía que se ocupa de la comprensión y correcta interpretación de la realidad. En este texto se reconoce su carácter epistemológico, no obstante, se recurre a su construcción como metodología para el acceso al trabajo de campo. 
Tabla 2. Núcleos problemáticos.

\begin{tabular}{|c|c|c|c|}
\hline $\begin{array}{l}\text { Núcleo } \\
\text { problemático }\end{array}$ & Subnúcleos & Referentes & Propósitos \\
\hline \multirow[t]{5}{*}{$\begin{array}{l}\text { 1. Socio- } \\
\text { profesional }\end{array}$} & 1.1. Personales & $\begin{array}{l}\text { - Género } \\
\text { - Edad }\end{array}$ & \multirow{3}{*}{$\begin{array}{l}\text { Conocer quiénes } \\
\text { son los estudiantes } \\
\text { del doctorado } \\
\text { en Educación }\end{array}$} \\
\hline & $\begin{array}{l}\text { 1.2. Contexto de } \\
\text { procedencia }\end{array}$ & $\begin{array}{l}\text { - Lugar de procedencia (entidad) } \\
\text { - Región en el estado de Guerrero }\end{array}$ & \\
\hline & 1.3. Contexto laboral & $\begin{array}{l}\text { - Nivel educativo en el que trabaja } \\
\text { - Sostenimiento de la escuela: pública/privada/otra } \\
\text { - Función } \\
\text { - Antigüedad laboral }\end{array}$ & \\
\hline & $\begin{array}{l}\text { 1.4. Formación } \\
\text { profesional }\end{array}$ & $\begin{array}{l}\text { - Licenciatura } \\
\text { - Maestría } \\
\text { - Doctorado } \\
\text { - Otros }\end{array}$ & \multirow[t]{2}{*}{$\begin{array}{l}\text { Analizar la formación } \\
\text { profesional de origen, } \\
\text { así como el campo en } \\
\text { el cual se desarrollan } \\
\text { profesionalmente }\end{array}$} \\
\hline & $\begin{array}{l}\text { 1.5. Ejercicio profesional } \\
\text { 1.6. Motivación por el } \\
\text { campo profesional }\end{array}$ & $\begin{array}{l}\text { - Identidad profesional en educación } \\
\text { - Otro campo profesional } \\
\text { - Factores positivos que orientan su incursión en educación } \\
\text { - Factores negativos que impactan en su incursión en educación }\end{array}$ & \\
\hline \multirow{2}{*}{$\begin{array}{l}\text { 2. Experiencia en } \\
\text { procesos de } \\
\text { investigación }\end{array}$} & 2.1. Formas de titulación & $\begin{array}{l}\text { - Modalidad de titulación en licenciatura } \\
\text { - Modalidad de titulación en maestría }\end{array}$ & \multirow{2}{*}{$\begin{array}{l}\text { Identificar la } \\
\text { experiencia previa } \\
\text { en materia de } \\
\text { investigación }\end{array}$} \\
\hline & 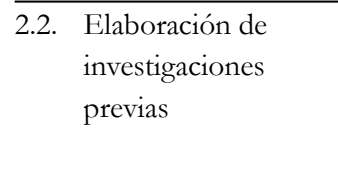 & $\begin{array}{l}\text { - Participación como responsable } \\
\text { - Participación como colaborador } \\
\text { - Instancia en la que desarrolló la investigación } \\
\text { - Finalidad de la investigación }\end{array}$ & \\
\hline \multirow[t]{5}{*}{$\begin{array}{l}\text { 3. Posicionamiento } \\
\text { epistemológico }\end{array}$} & $\begin{array}{l}\text { 3.1. Formación } \\
\text { epistemológica }\end{array}$ & $\begin{array}{l}\text { - Cursos de epistemología en estudios formales (curricular) } \\
\text { - Cursos de epistemología externos al currículo }\end{array}$ & \multirow{5}{*}{$\begin{array}{l}\text { Analizar los factores } \\
\text { que conforman } \\
\text { la identidad } \\
\text { epistemológica de } \\
\text { los investigadores en } \\
\text { formación }\end{array}$} \\
\hline & $\begin{array}{ll}\text { 3.2. } & \text { Relación entre } \\
& \text { objeto de estudio } \\
\text { y contexto laboral }\end{array}$ & $\begin{array}{l}\text { - Relación consistente } \\
\text { - Relación parcial } \\
\text { - No existe relación }\end{array}$ & \\
\hline & $\begin{array}{l}\text { 3.3. Posicionamiento } \\
\text { epistemológico }\end{array}$ & $\begin{array}{l}\text { - Escuela epistemológica que soporta las intenciones de } \\
\text { generación de conocimiento } \\
\text { - Intenciones de generación de nuevos conocimientos (intenciones } \\
\text { para hacer investigación) }\end{array}$ & \\
\hline & $\begin{array}{l}\text { 3.4. Posicionamiento } \\
\text { metodológico }\end{array}$ & $\begin{array}{l}\text { - Paradigma de investigación } \\
\text { - Diseño del estudio con relación al paradigma seleccionado } \\
\text { - Relación entre: posicionamiento epistemológico/paradigma de } \\
\text { investigación/diseño del estudio/campo de formación disciplinar } \\
\text { - Intenciones de uso de las técnicas de investigación } \\
\text { - Valores que orientan el sentido ético de su rol como investigador }\end{array}$ & \\
\hline & $\begin{array}{l}\text { 3.5. Difusión del } \\
\text { conocimiento }\end{array}$ & $\begin{array}{l}\text { - Elaboración de artículos } \\
\text { - Elaboración de ponencias } \\
\text { - Otros productos }\end{array}$ & \\
\hline \multirow{2}{*}{$\begin{array}{l}\text { 4. Idea fuerza } \\
\text { del futuro } \\
\text { académico }\end{array}$} & $\begin{array}{l}\text { 4.1. Proyección de su } \\
\text { ámbito profesional }\end{array}$ & - Acciones en el futuro profesional & \multirow{2}{*}{$\begin{array}{l}\text { Identificar los puntos } \\
\text { de énfasis sobre su } \\
\text { futuro como } \\
\text { investigadores }\end{array}$} \\
\hline & $\begin{array}{ll}\text { 4.2. } & \text { Proyección de su rol } \\
& \text { como investigador }\end{array}$ & - Acciones en el futuro en carácter de investigador & \\
\hline
\end{tabular}


Tabla 3. Sujetos de investigación.

\begin{tabular}{|c|c|c|c|c|c|c|}
\hline No. & Género & Edad & $\begin{array}{l}\text { Entidad de } \\
\text { procedencia }\end{array}$ & Nivel en que labora & Función & $\begin{array}{c}\text { Antigüedad } \\
\text { laboral }\end{array}$ \\
\hline 1 & Hombre & 59 & Morelos & Licenciatura & Docente investigador & 36 años \\
\hline 2 & Mujer & 54 & Ciudad de México & Licenciatura & Docente & 20 años \\
\hline 3 & Hombre & 46 & Guerrero & Secundaria y maestría & Docente & 14 años \\
\hline 4 & Mujer & 35 & Guerrero & Primaria & Docente & 14 años \\
\hline 5 & Mujer & 35 & Guerrero & Secundaria & Jefa de área & 12 años \\
\hline 6 & Mujer & 35 & Guerrero & Maestría & Docente investigadora & 5 meses \\
\hline 7 & Mujer & 34 & Guerrero & Licenciatura y maestría & Docente y jefe de área & 7 años \\
\hline 8 & Mujer & 27 & Guerrero & Secundaria & Docente & 4 años \\
\hline
\end{tabular}

Fuente: Construcción personal.

Atendiendo a la tabla 3, se observa que el $75 \%$ de los entrevistados son mujeres y también el 75\% son originarios del estado de Guerrero, aunque el 100\% radica en el Puerto de Acapulco. Las edades oscilan entre los 27 y los 59 años, teniendo una media de 35 años. El 100\% labora en el campo de la educación, desarrollando funciones de docencia y coordinación académica. En cuanto al total que ejerce la docencia en el nivel superior, lo hace en programas de licenciatura y posgrado del área de educación y ciencias biológicas en la Universidad Autónoma de Guerrero (UAGro), en el Instituto Tecnológico de Acapulco (ITA) y en el Instituto de Estudios Universitarios (IEU).

A través de la entrevista se accedió a información de los cuatro núcleos problemáticos, pudiendo enfatizar los factores que conforman su identidad epistemológica como investigadores, aunque en este trabajo son abordados con mayor énfasis los aspectos relacionados al segundo y tercer núcleos. Las entrevistas fueron registradas en formato doble: grabación de voz - autorizada por los sujetos de estudio- y captura en texto. El diálogo desarrollado permitió la comprensión e interpretación de sus palabras bajo los principios hermenéuticos, no obstante, como recurso complementario fue empleado el software MaxQDA, en este se registró el sistema de núcleos problemáticos y fueron procesadas las entrevistas.

El análisis desarrollado permitió obtener matrices de fragmentos con los hallazgos generados en cada categoría hermenéutica, lo que a su vez posibilitó derivar puntos de consistencia e inconsistencia. Los fragmentos recuperados fueron revisados mediante su posicionamiento histórico y lingüístico. Se buscó construir sentido, no solo con relación a las palabras centrales que distinguieron la opinión de los entrevistados, sino también considerando el sentido global obtenido respecto al marco temático. Conforme a la lectura de las entrevistas y siguiendo la estructura planteada en la definición de núcleos problemáticos fueron recuperados los fragmentos con mayor 
carga de sentido, tanto coincidentes como discrepantes, posteriormente se procedió a establecer un debate entre ambos referentes, dando lugar a la construcción de sentido a los datos, misma que llevó a la presentación y discusión de los resultados.

\section{Resultados Y Discusión}

En el siguiente apartado se encuentran los resultados obtenidos tras el diálogo con los entrevistados; en particular se hace referencia a los apartados 1.5 y 1.6 del primer núcleo problemático, así como al total del segundo y tercer núcleos, sobre los cuales se construyeron las intenciones de comprender quiénes son los estudiantes del doctorado en Educación y cuáles son los factores involucrados en la conformación de su identidad epistemológica.

La revisión de las propuestas curriculares de los principales programas de doctorado ubicados en la zona sur de la FIMPES permite afirmar que la formación epistemológica de los estudiantes suele estar acotada a los cursos iniciales, destacando un posterior énfasis en la dimensión metodológica. Esta situación es consistente con el plan de estudios que cursaron los entrevistados, ya que también al inicio de su formación abordaron las bases epistemológicas de la investigación y posteriormente se privilegió la discusión metodológica.

En lo concerniente a la decisión de formarse a nivel doctoral, prevaleció la intención de trascender ontológicamente, para sí mismos y para construir nuevas formas de realidad educativa y social en el contexto que los ocupa. Guerrero es una entidad que en la segunda década del siglo XXI se ha visto impactada por la violencia, y en el caso particular del Puerto de Acapulco, a principios del año 2017 el Consejo Ciudadano para la Seguridad Pública y Justicia Penal lo ubicó como la segunda ciudad más violenta del mundo. En este marco, los doctorantes afirmaron haber asumido sus estudios como una oportunidad para transformar su entorno desde un posicionamiento dotado de rigor académico y consciencia social. En este sentido, un entrevistado mencionó:

Estudié el doctorado porque es un sueño, sin embargo, en el camino descubrí que a través de la investigación se puede hacer algo por nuestra realidad que se encuentra marcada por la violencia y el rezago educativo [E8F2].

Otro propósito de formación derivó de la comprensión de los tiempos políticos establecidos en la agenda educativa nacional, y en particular de la interpretación de los tiempos institucionales que se ven impactados por esta en cada entorno laboral de los entrevistados. Sobre este punto se comentó lo siguiente:

Hace, no sé, diez o quince años se ha venido impulsando la necesidad de que particularmente los tiempos completos nos involucrásemos más con el área de investigación, entonces, al principio no le entendíamos a eso y en este momento mi interés consiste en entender lo que estoy 
haciendo, entender por qué lo hago y yo pienso que esas respuestas están en la teoría, por eso hay que entrar en la investigación; por qué estoy haciendo, para qué estoy haciéndolo [E2F6].

El interés laboral constituyó un motivo para la apropiación de las políticas que han impactado su condición profesional y la configuración de su perfil académico. Por un lado está un sector para quienes la incursión en el mundo de la investigación resultó un claro aliciente para atender las obligaciones conferidas a su categoría laboral, pero la disposición a formarse y ejercer esta labor trascendió lo normativo y colocó a los doctorantes en la posibilidad de ascenso hacia un estatus de supremacía académica respecto a otras categorías laborales y otros niveles de habilitación profesional; por otro lado está el sector de quienes se posicionaron más allá de la encomienda que se confiere a una categoría laboral. No obstante, en la estructura organizacional de sus instituciones, formarse en el nivel doctoral también implicó el acceso a nuevos imaginarios sobre calidad académica.

En cuanto a la decisión de matricularse en una institución privada, prevaleció una narrativa que cobró énfasis en la educación superior durante la década de 1970, se trata de la masificación y entorno politizado. Esta época se caracterizó por la masificación del sector que accedió a la educación superior pública, aunque el crecimiento cuantitativo no se correspondió de forma cualitativa, lo que impactó en la disminución de la calidad de la oferta educativa. Esta situación derivó en que determinado sector con mayores posibilidades económicas ingresara en instituciones privadas, no obstante, en la entidad no se instalaron ofertas de élite, sino las reconocidas por Muñoz, Núñez y Silva (2004) como de "absorción de la demanda", las cuales, considerando las variables de tiempo y tamaño, están divididas en "consolidadas" y "en consolidación" y "emergentes". Al respecto, otro entrevistado señaló:

La Universidad Autónoma de Guerrero siempre ha sido la primera opción, pero no ofrece un doctorado que directamente sea de educación, me parece que tiene uno en enseñanza de las matemáticas, pero no es el tema que me interesa. Hay otro en otra escuela pública, pero como casi todo lo que está en el estado, se caracteriza por su carácter politizado y no por su calidad, es más, por lo mismo, sus alumnos son sus propios maestros que trabajan ahí porque de fuera no llegan. En cuanto a las privadas [...] esta fue la mejor opción, tanto por su modelo como por sus profesores [E4P17].

Los entrevistados fueron enfáticos en señalar que el IEU, teniendo en consideración la oferta total de universidades privadas que se encuentran en Acapulco, fue su mejor opción, pudiendo estar colocada como una universidad de absorción de la demanda, pero consolidada con más de 40 años de historia, lo que constituye un referente de buena calidad. En cuanto al contexto politizado, todos afirmaron que el Estado no ha logrado diversificar la oferta educativa de calidad; es así como, en el sector público, existen instituciones con prestigio que no cuentan con una oferta de doctorado directa en el campo de la educación, y en las que sí lo ofertan prevalece 
un clima político intenso que llevó a buscar una opción reconocida por su calidad académica entre la oferta de corte privado que llegó al sur del país.

En cuanto a su formación previa, estudiaron maestrías profesionalizantes, en las cuales, además de la opción de tesis, el grado también se puede obtener por actualización extracurricular, teniendo un promedio final igual o superior a nueve o, en su defecto, mediante la elaboración de un proyecto enfocado a la atención de problemas institucionales, por lo regular construidos desde el marco de la gestión educativa. En este sentido, un entrevistado comentó:

En la maestría sacaba buenas calificaciones y en el doctorado me di cuenta de que la calificación es lo de menos, comprendí que no era lo esencial. En la maestría buscábamos el mejor promedio para titularnos sin tener que hacer tesis ya que así lo permite la universidad, por eso, mi única experiencia en investigación cuando entré fue mi tesis de licenciatura [E8F5].

Por eso, la participación en procesos de investigación previos a su condición de estudiantes de doctorado resultó breve y en el mejor de los casos se encontró relacionada a la elaboración de estudios de ascenso académico, situación que condicionó su experiencia en investigaciones que debieron dotarlos de habilidades propicias para la generación de nuevos conocimientos. Aun así, el posicionamiento epistemológico está presente en el trabajo de todos los investigadores aún y cuando en un principio no logran argumentar su presencia en sus propias narrativas. En este marco y dadas las circunstancias revisadas, se percibió un capital epistemológico en conformación que limitó la posibilidad de argumentar la importancia que esta dimensión tiene para la investigación. En esta línea de análisis fue planteado lo siguiente:

Fue relativa la importancia que atribuimos a la epistemología para el desarrollo de nuestra investigación. Se cursaba como una materia en la cual se hacía referencia a su objetivo de estudiar cómo se genera el conocimiento [...] pero no se pasaba a analizar situaciones en las cuales pudieras comprender a qué se refería o cómo se hacía o podría ocuparse en nuestros trabajos de investigación [E3F9].

En su proceso formativo, el análisis de la epistemología constó de un curso en el que se emprendió una revisión teórica que desde el inicio posicionó un énfasis metodológico, sin que se alcanzara a argumentar con dominio experto cómo este forma parte de la base epistemológica que les debe permitir afirmar por qué sus hallazgos pueden ser considerados como conocimiento y no como cualquier otra cosa (Llano, 1984). Es decir, fundamentar que la metodología constituye la estructura, el aparato de facto que permite hacer posibles las intenciones de su estudio establecidas en la fase epistémica. En este sentido, otro entrevistado mencionó:

Existe una línea de formación perfectamente establecida en términos metodológicos, o sea, desde el inicio te dejan claro, tanto en los recursos de consulta como en el discurso de la mayoría de los doctores, que tienes que encontrar un problema que tenga relación con tu contexto laboral y a través de una investigación buscar conocerlo y resolverlo, generar propuesta para la mejora, 
cuantitativos, cualitativos y mixtos. Lo mixto es lo que recomiendan con mayor énfasis y de ahí el que sigue en importancia es el cuantitativo. Este está muy marcado en el diseño de los cursos y en el discurso de los profesores [E5P12].

Además de constituir el referente de énfasis en los procesos formativos, también fue posible percatarse de la tradición institucional. Conforme a esta, prevaleció una visión cuantitativa próxima a las escuelas Positivista y Empírica, tanto en el diseño de los cursos como en el capital académico de los profesores, en quienes también predominó un discurso similar. Así se construyó una didáctica de la investigación que los estudiantes se apropiaron desde el principio, y aunque para algunos sus intereses de estudio se colocaron en la vertiente cualitativa cercana a las escuelas Fenomenológica y Hermenéutica, en su experiencia cotidiana prevaleció una narrativa cuantitativa que, como base de incursión a la investigación, impactó en la configuración de su identidad como investigadores.

En cuanto a la voz de los actores que conformaron la planta académica, en la mayoría de ellos no solo destacó la consistencia de su posicionamiento epistemológico con la tradición institucional, sino todavía más: los estudiantes encontraron en ellos referentes de identidad. Es decir, al compartir su visión sobre la investigación, así como los productos de su labor como investigadores, influyeron en su posicionamiento académico, en la selección de su aparato metodológico, así como en la generación de expectativas de éxito sobre su propio futuro como investigadores.

Además de este punto, el fragmento revisado dejó ver otros referentes de análisis, entre estos la relación entre el objeto de estudio y el contexto laboral, así como la identidad docente. En este sentido destacó una narrativa conforme a la cual se asume que la investigación en el campo educativo tiene la intención de resolver los problemas que son detectados en el contexto empírico. Así, la selección de sus temas se soportó principalmente en su experiencia educativa, en lo que percibieron en sus centros laborales como puntos de tensión que demandaban ser resueltos para restablecer la armonía institucional. Al respecto, otro entrevistado señaló:

Te piden que investigues un problema y propongas cómo resolverlo, así que revisas los muchos que tienes en la escuela y los consejos técnicos se convierten en una fuente que te sirve mucho [E6P14].

En consecuencia, uno de los primeros retos que enfrentaron consistió en saber diferenciar entre el objeto de estudio de la gestión y el de la investigación; en argumentar que la investigación está orientada hacia la generación de nuevos conocimientos o nuevas interpretaciones al conocimiento existente con el rigor epistemológico, teórico y metodológico necesario para ser aceptados en la comunidad académica, lo cual, en suma, constituye una aportación al propio estado del conocimiento y a la comprensión del referente empírico. 
En referencia a otro punto, algunos entrevistados cuentan con una formación de origen situada en el campo de la educación y otro sector proviene de las ciencias sociales. No obstante, todos incursionaron laboralmente en el sistema educativo, tanto en escuelas públicas como privadas, algunos en el nivel básico, otros en el medio superior y superior. Esto implica que por formación profesional o por profesionalización en la práctica han construido su identidad docente, lo que, además de la experiencia, constituye un filtro pedagógico, ideológico y político que les debe permitir comprender los múltiples objetos de estudio que pueden encontrar en la educación. Sin embargo, cuando buscaron hacer explícitos los ejes que articulan su identidad docente esta se mostró dispersa, destacando también la ausencia de la base epistemológica desde la cual construyen sentido a la realidad; en cambio, enfatizaron la intención gestora de su labor. En el siguiente párrafo uno de los entrevistados dejó ver su experiencia:

El campo educativo no fue mi primera opción, pero la vida te depara muchos caminos y te abre puertas que en ciertos momentos no puedes dejar pasar, entonces, te encuentras en el dilema de seguir buscando o tomarlas y pues yo la tomé y al inicio no fue fácil, te haces docente en la práctica y abrazas la profesión y primero retomas modelos de gente que conoces, hasta que logras decir "así soy yo", y aún así, reconozco carencias sobre mi ser docente que todavía no planteo completamente [E3F18].

Además se reconoció la presencia de la ideología como eje articulador de la identidad que construyen como investigadores. Se trata de un posicionamiento que asumen teniendo como génesis su propia historia de vida, las circunstancias de sus contextos sociales, las herencias culturales, pero también el carácter social que asumen las instituciones en las cuales se formaron y en las cuales laboran. Otro entrevistado planteó lo siguiente respecto a este punto de discusión:

Cuando estaba en la fase documental pude percatarme de que los textos no son neutros, al menos los que no son netamente teóricos, todos toman un lado desde el cual ven el tema o cómo este se percibe en la realidad y también suma la parte de las escuelas en las que te formas, las públicas de la montaña perciben algo muy distinto de las públicas del puerto, y de las privadas ni hablar, por eso digo que abordas la investigación sabiéndote parte de un contexto, pero también influido por los lugares en los cuales te preparaste profesionalmente [E7F22].

Es posible percatarse de que la ideología siempre ha estado presente en la forma en que asumen su labor educativa y también al posicionarse frente a su objeto de estudio y frente a la realidad en la cual lo indagan. En la opinión de los entrevistados, la ideología se muestra abiertamente en su contexto, el cual se ha caracterizado por su historia altamente politizada y por la confrontación permanente entre grupos hegemónicos y disidentes que se posicionan en el gobierno local.

Estamos en Guerrero, somos un lugar de lucha, un lugar del sur que se mide aparte con relación al resto del país. Siempre estamos en un lugar con relación a otro, siempre existe una lucha por el poder o por no verse oprimido por el poder, una lucha de ideales, y la escuela no es ajena a esa lucha, aunque muchos compañeros profesores parecen no enterarse de su existencia [E2F13]. 
Como investigadores, señalaron que se ven siempre impactados por la historicidad sobre la cual han sido formados, por la tradición que permea la idiosincrasia de sus instituciones formadoras, por la construcción dialéctica que se sostiene entre el pasado y presente de su propio contexto, siempre en debate sobre lo que es, lo que ha sido y lo que puede ser. El futuro como posibilidad los ha llevado a posicionarse en un frente ideológico desde el cual se colocan para luchar por su construcción, por ende, siempre encuentran en la vida social y en la vida escolar un espacio de lucha política, un frente de lucha ideológica al cual no escapa la formación que han recibido, la que reciben sus alumnos y, por ende, la forma en que ejercen su rol como investigadores.

Reflexionando sobre su futuro, afirmaron que la base ontológica y el compromiso social que motivó sus estudios conforman los dos grandes ejes sobre los cuales posicionarán su desempeño académico, sin embargo no descartaron la posibilidad de encontrar en su grado un motivo de reconocimiento y ascenso académico y, si es posible, laboral. Uno de los puntos de vista comentados es el siguiente:

Me queda claro que tengo mucho que aprender, tengo que madurar mi pensamiento, tengo que madurar mi discurso teórico y metodológico, se me tiene que notar lo doctor en lo que pienso y en lo que digo. Esto es el inicio, la tesis solo es el inicio, pero lo asumo con seriedad, con rigor. No busco un título por imagen, si este me ayuda en lo laboral bienvenido, sin embargo, lo que me importa ahora es seguirme formando como un verdadero investigador, así que debo seguir leyendo y escribiendo para continuar comprendiendo y transformando mi contexto empírico con alto rigor académico [E1F16].

En este marco, tomando en cuenta los conceptos de adscripción y afiliación desarrollados por Gil, Grediaga, Pérez, Rondero, Casillas y De Garay (1994), también es posible percatarse de las implicaciones de los estudios doctorales en el proceso de incorporación a la comunidad académica. En principio trajo consigo la posibilidad de fortalecer su adscripción laboral, lo que concierne a la pertenencia a una institución y a la categoría laboral con la cual ejercen su función, formalizada a través de un contrato. En este caso, para quienes laboran en educación básica, el doctorado los sometió a una doble tensión: por un lado se construyeron mayores imaginarios de calidad sobre su formación y, en consecuencia, mayores expectativas sobre su desempeño, incluso en lo que no concierne a la investigación; por otra parte los habilitó para buscar escenarios de ascenso en la estructura institucional, pero al mismo tiempo les generó mayores méritos para incorporarse a la educación superior ejerciendo la docencia e investigación.

En cuanto a la afiliación, esta corresponde a la pertenencia al campo de estudio en el cual se han formado. Todos los entrevistados coincidieron en que el doctorado legitimó su incorporación al grupo cerrado de quienes se asumen como investigadores en su contexto. Definitivamente, el correcto equilibrio entre la adscripción y la afiliación debe conformar una base no solo para incorporarse al grupo académico, sino 
fundamentalmente para construir una trayectoria exitosa, lo que, sin duda, también esperan que impacte en la mejora de sus condiciones de trabajo, en la posibilidad de centrar su quehacer en actividades medulares que los lleven a buenos niveles de producción académica y a enfrentar la realidad en la cual están insertos, contribuyendo a su transformación desde la academia.

\section{Modelo para el análisis de la identidad epistemológica de los doctorantes en Educación}

Tomando en cuenta las aportaciones de los doctorantes, así como la obra de Zizek (2003), se propone un modelo de identidad epistemológica que puede ser implementado para formar a los investigadores en educación. Este se compone por un punto nodal que cohesiona y dota de sentido a cuatro significantes flotantes: sistema de lenguaje, posicionamiento ideológico, contexto histórico-cultural y posicionamiento docente.

El punto nodal corresponde a la noción de escuelas epistemológicas, es decir, el investigador se posiciona sobre una de las escuelas que explican la génesis del conocimiento; implica comprender la naturaleza de la investigación y de cómo emprenderla. En esta etapa se reconoce que al hacer investigación el punto de partida siempre debe radicar en la definición de las intenciones de generación de conocimiento, ya que cuando son planteadas con rigor académico será posible construir el aparato instrumental llamado "metodología". El posicionamiento relacionado con las escuelas epistemológicas puede considerar dos extremos como referencia: en uno se encuentra la positivista-empirista y en otro la fenomenológica-hermenéutica.

Los significantes flotantes son el sistema de lenguaje, el posicionamiento ideológico, el contexto histórico-cultural y el posicionamiento docente.

- Sistema de lenguaje: el lenguaje constituye el medio en el que se realiza el acuerdo entre los interlocutores mediante el diálogo. El lenguaje en sí mismo refleja ya una concepción del mundo (Morales, Benavides, Reséndiz, Haro, Franco y Dixon, 2012), por eso el esclarecimiento de la escuela epistemológica implica para el investigador la necesidad de construir sus argumentos teóricos y metodológicos, fundamentados en un lenguaje propio de la base seleccionada. Este hecho evitará que incurra en contradicciones en el desarrollo de sus narrativas, así como en la construcción de sentido a los datos que conforman la base de sus hallazgos. Implica el manejo de un lenguaje especializado desde el cual se debe leer, escribir y dialogar con la realidad.

- Posicionamiento ideológico: la ideología, siguiendo la propuesta de Sánchez (1993), tiene que ver con el conjunto de ideas acerca del mundo y la sociedad, las cuales responden a intereses, aspiraciones o ideales de una clase social en un contexto dado, el cual orienta y justifica el comportamiento práctico de 
los seres humanos conforme a esos intereses o ideales. Por eso resulta preciso reconocer que la investigación no se construye al margen de la ideología, al contrario, esta coloca el punto de partida desde el cual se justifica la realidad, asumiéndose en el marco de ideas de un grupo social, definiendo los bordes de la conciencia sobre esta, no solo para enfatizarlos sino incluso para cuestionarlos. En este marco es posible situar como referentes de discusión a dos posturas sociales: tecnogerencia y teoría crítica.

- Contexto histórico-cultural: el investigador no solo está construido por su autopercepción sino también por las herencias culturales y formas simbólicas que le permiten identificarse con un contexto; a juicio de los expertos, estos se mantienen presentes en la forma en que se toma contacto con el mundo. En cuanto al tiempo, todo proceso de investigación se sujeta a una temporalidad, sin embargo los objetos de estudio que son indagados no necesariamente son producto de una perspectiva inmediata, por el contrario, la trascienden recuperando lo pasado que pertenece a la tradición, por lo que al indagar la realidad también se está elaborando un proceso de recuperación del tiempo que compromete al investigador a comprender la tradición sobre la cual se posiciona.

- Posicionamiento docente: incursionar como investigador en el campo educativo, siendo parte de este, requiere de estar conscientes de la propia identidad docente, la cual permite asumir posiciones de diálogo con el contexto y objeto de estudio que se indaga, por eso, siguiendo la misma base de diseño soportada en un punto nodal y sus significantes flotantes, en la tabla 4 se muestran dos formas en que el investigador puede posicionarse como docente para contribuir a la revisión y construcción del sistema educativo, aunque resulta necesario reconocer que entre ambos extremos existen puntos de encuentro a considerar en su labor profesional.

Conforme al modelo planteado, se reconoce el carácter hermenéutico que existe en la conformación de la identidad epistemológica, es decir, esta constituye un ente abierto, un proyecto que se nutre del conocimiento y tradición a las cuales se enfrenta el investigador en la interacción con las herencias institucionales y con la idiosincrasia epistemológica de sus formadores. Esta tesis implica asumir que la identidad se irá consolidando en tanto logre comprender e interpretar esas tradiciones en el marco de la definición de su objeto de estudio. La identidad epistemológica se manifiesta en la narrativa que los estudiantes construyen de sí mismos, en la comprensión e interpretación de sus intenciones de generación de conocimientos frente a una realidad que los reta a conocerla y transformarla, por ende, la identidad se devela en la autointerpretación de los motivos de la incursión al campo de la investigación, lo cual se construye haciendo uso del relato de sí mismos. 
Tabla 4. Dos formas de concebir la identidad del profesor.

\begin{tabular}{|c|c|c|}
\hline $\begin{array}{l}\text { Significante } \\
\text { flotante }\end{array}$ & $\begin{array}{c}\text { Tesis a } \\
\text { (Tecnogerencial) }\end{array}$ & $\begin{array}{l}\text { Tesis b } \\
\text { (Teoría crítica) }\end{array}$ \\
\hline $\begin{array}{l}\text { Idea de } \\
\text { ser humano }\end{array}$ & $\begin{array}{l}\text { Sujeto instrumental cuyo fin radica en } \\
\text { reproducir un modelo de sociedad }\end{array}$ & $\begin{array}{l}\text { Ser consciente de sí mismo que se encuentra en la búsqueda } \\
\text { permanente del fortalecimiento de su condición humana }\end{array}$ \\
\hline $\begin{array}{l}\text { Concepto de } \\
\text { educación }\end{array}$ & $\begin{array}{l}\text { Habilitación académica e instrumental con fines } \\
\text { utilitarios que permitan al capital humano } \\
\text { funcionar con eficacia ante las expectativas que } \\
\text { se trazan de sí mismo y de su aporte social }\end{array}$ & $\begin{array}{l}\text { Proceso hermenéutico en el cual, la oportunidad de conver- } \\
\text { sar con uno mismo, con los otros y con el contexto, con- } \\
\text { forman la base para educarse y para transformar la realidad } \\
\text { haciendo uso de conocimientos, habilidades y valores con } \\
\text { sentido humano }\end{array}$ \\
\hline Teorías sociales & Tecnogerencial & Teoría crítica \\
\hline $\begin{array}{l}\text { Posicionamiento } \\
\text { pedagógico }\end{array}$ & $\begin{array}{l}\text { Pedagogías diversas orientadas hacia fines } \\
\text { utilitaristas }\end{array}$ & $\begin{array}{l}\text { Pedagogía crítica } \\
\text { (pedagogía de la liberación) }\end{array}$ \\
\hline $\begin{array}{l}\text { Concepto de } \\
\text { enseñanza }\end{array}$ & $\begin{array}{l}\text { Proceso de capacitación bajo el estricto } \\
\text { dominio del profesor y sin considerar } \\
\text { la participación del estudiante }\end{array}$ & $\begin{array}{l}\text { Diálogo abierto, en el cual, el profesor abandona la acción } \\
\text { de acumular en el estudiante paquetes de conocimiento, } \\
\text { para entonces, promover una actitud de encuentro entre } \\
\text { seres humanos }\end{array}$ \\
\hline $\begin{array}{l}\text { Concepto de } \\
\text { aprendizaje }\end{array}$ & $\begin{array}{l}\text { Acumulación de conocimientos con la intención } \\
\text { de instrumentarlos en la realidad }\end{array}$ & $\begin{array}{l}\text { Actitud de diálogo y disposición a la acción que permita la } \\
\text { construcción de conocimientos y experiencias orientadas al } \\
\text { despertar de la consciencia de sí mismo y en comunidad, } \\
\text { para comprender la realidad y transformarla }\end{array}$ \\
\hline $\begin{array}{l}\text { Idea de } \\
\text { evaluación }\end{array}$ & Proceso de medición con fines instrumentales & $\begin{array}{l}\text { Proceso que equilibra aspectos cuantitativos y cualitativos } \\
\text { para comprender la realidad y promover pautas de mejora } \\
\text { permanente }\end{array}$ \\
\hline $\begin{array}{l}\text { Noción de } \\
\text { calidad }\end{array}$ & $\begin{array}{l}\text { Isomorfismo o de la calidad soportada } \\
\text { en acreditaciones y certificaciones diversas }\end{array}$ & $\begin{array}{l}\text { Estado de desarrollo positivo con potencial de mejora } \\
\text { permanente en su condición cuantitativa y cualitativa }\end{array}$ \\
\hline $\begin{array}{l}\text { Referente } \\
\text { Educativo } \\
\text { Nacional }\end{array}$ & $\begin{array}{l}\text { Poco reconocimiento a actores nacionales } \\
\text { que han contribuido al desarrollo del } \\
\text { proyecto educativo }\end{array}$ & $\begin{array}{l}\text { Reconocimiento pleno de actores nacionales que han } \\
\text { contribuido al desarrollo de un proyecto político-educativo } \\
\text { (Sierra, Vasconcelos, Barreda, Ramírez, Rébsamen, Torres } \\
\text { Bodet, etc.) }\end{array}$ \\
\hline
\end{tabular}

Fuente: Construcción personal.

\section{Conclusiones}

Estudiar un doctorado implica formarse como investigador y la tarea de los investigadores; si bien abarca el ejercicio de la docencia con alto rigor académico, principalmente se orienta a la generación de nuevos conocimientos o a la construcción de nuevos sentidos al conocimiento existente, por lo que se requiere de una formación epistemológica rigurosa ya que esta constituye la dimensión que le permitirá argumentar porqué sus hallazgos deben ser considerados como conocimientos.

En este sentido se reconoce que los estudiantes cuentan con una identidad epistemológica parcialmente articulada, la cual, al desarrollar sus estudios, se acota a una predisposición metodológica (cuantitativa-cualitativa-mixta). Por lo tanto, el 
principal reto que enfrentan consiste en la definición de las intenciones de generación de conocimiento, lo que solo es posible mediante la consolidación de su identidad epistemológica, la cual incluso se soporta en la dimensión ontológica que precisa la idea de ser humano que se construye en su investigación.

Los estudiantes cuentan con distintos niveles de conciencia respecto a la conformación de su identidad docente. Algunos privilegian una visión instrumental de la educación, mientras otros han accedido a su naturaleza humanista y política. En consecuencia, se requiere de la plena articulación entre su identidad docente y epistemológica para lograr construir sentido correcto a sus hallazgos, los cuales, solo así, estarán soportados en el uso de una metodología rigurosa que, como factor de éxito, considere la comprensión y reconocimiento de lo complejo del campo educativo en acción.

En definitiva, se reconoce que el trabajo de investigación no se construye al margen de la tradición del contexto de estudio, por lo que siempre existe un posicionamiento ideológico-social desde el cual el investigador se coloca para indagar la realidad. Incluso las instituciones en las cuales se ha formado y en las que labora se asumen en una vertiente ideológica que, entendida en los extremos, se ubican entre las vertientes tecnogerencial y la teoría crítica. Por ende, se requiere de analizar y reconocer esta dimensión con mayor fortaleza en el diseño de todo trabajo de investigación, siempre que esté acompañada de un diálogo permanente con la dimensión ética.

Para el diseño del modelo de identidad epistemológica se partió del concepto de Zizek (2003), quien señaló que la identidad se compone de un punto nodal (escuela epistemológica) que articula a los significantes flotantes (sistema de lenguaje, posicionamiento ideológico, contexto histórico-cultural y posicionamiento docente), sin embargo el mayor de los fundamentos se encontró en la voz de los estudiantes, quienes, desde su experiencia, asumieron una disposición hermenéutica soportada en una narrativa de sí mismos, compartiendo ejes trascendentales que impactaron su formación doctoral, mismos que han sido incorporados en esta propuesta, la cual se espera que contribuya para habilitar investigadores con una formación académica rigurosa en los programas de maestría y doctorado en Educación.

\section{REFERENCIAS}

Álvarez, G. (2002). La calidad y la innovación en los posgrados. Revista de la Educación Superior, (124), 31-38. Recuperado de: http://publicaciones.anuies.mx/pdfs/revista/ Revista124_S3A2ES.pdf.

Briones, G. (2002). Epistemología de las ciencias sociales. Recuperado de: https:/ /www.insumisos.com/lecturasinsumisas / Epistemologia\%20de\%20las\%20ciencias\%20sociales.pdf.
Campos-Winter, H. (2018). Estudio de la identidad cultural mediante una construcción epistémica del concepto identidad cultural. Cinta Moebio, (62), 199-212. Doi: 10.4067/S0717-554X2018000200199.

Castro, L., Castro, M., y Morales, J. (2016). Metodología de las ciencias sociales. Una introducción crítica (3a. ed.). Madrid: Tecnos. 
Fernández, L., y Wainerman, C. (2015). La dirección de tesis de doctorado: ¿una práctica pedagógica? Perfiles Educativos, 37(148), 156-171. Recuperado de: http://www. scielo.org.mx/pdf/peredu/v37n148/v37n148a10.pdf.

Fresán, M. (2002). La asesoría de la tesis de doctorado. Una influencia permanente en la vida del investigador independiente. Revista de la Educación Superior, 31(124), 103-122. Recuperado de: https://www.redalyc.org/ pdf/140/14003112.pdf.

Gadamer, H. (2000). La educación es educarse. España: Paidós.

Gadamer, H. (2001). Antología. España: Sígueme-Salamanca.

Gadamer, H. (2007). Verdad y método. Salamanca: Ediciones Sígueme.

García, J. (2009). Las políticas y los programas de posgrado en México. Una dinámica de contrastes entre 1988 y 2008. Sociológica, 24(70), 153-174. Recuperado de: http:// www.scielo.org.mx/pdf/soc/v24n70/v24n70a6.pdf.

Gil, M., Grediaga, R., Pérez, L., Rondero, N., Casillas, M., y De Garay, A. (1994). Los rasgos de la diversidad. Un estudio sobre los académicos mexicanos. México: UAM-Azcapotzalco.

Giménez, G. (2010). La cultura como identidad y la identidad como cultura. México: Instituto de Investigaciones Sociales de la UNAM.

Grondin, J. (2008) ¿Qué es la hermenéutica? España: Herder.

Hessen, J. (1982). Teoría del conocimiento (16a. ed.). México: Austral.

Kvale, S. (2011). Las entrevistas en investigación cualitativa. España: Morata.

Llano, A. (1984). Gnoseología. Pamplona: Universidad de Navarra.

Lora, J., y Sánchez, J. (2012). Hacia una metodología de la praxis. La investigación social en gráficas. Puebla: Instituto de Ciencias Sociales y Humanidades Alfonso Vélez Pliego, BUAP.

Mendoza, J. (2002). La transición de la educación superior contemporánea en México: de la planeación al estado evaluador. México: Fondo de Cultura Económica.

Möller, I., y Gómez, H. (2014). Coherencia entre perfiles de egreso e instrumentos de evaluación en carreras de educación básica en Chile. Calidad en la Educación, (41), 17-49. Recuperado de: https://scielo.conicyt.cl/pdf/ caledu/n41/art02.pdf.

Morales, B., Benavides, L., Reséndiz, M., Haro, K., Franco, L., y Dixon, E. (2012). La epistemología y la metodología. Un binomio fundamental para la construcción de la tesis. México: Benemérita Universidad Autónoma de Puebla.

Muñoz, C., Núñez, M., y Silva, M. (2004). Desarrollo y beterogeneidad de las instituciones de educación superior particulares. Colección Biblioteca Educación Superior. México: ANUIES.

Neave, G. (2001) Educación superior: historia y politica. Estudios comparativos sobre la universidad contemporánea. España: Gedisa.

Núñez-Valdés, K., y González, J. (2019). Perfil de egreso doctoral: una propuesta desde el análisis documental y las expectativas de los doctorados. IE Revista de Investigación Educativa de la REDIECH, 10(18), 161-175. Doi: https://dx.doi.org/10.33010/ie-rie-rediech.v10i18.604.

Osorio, F., Páramo, P., Otálvaro, G., Posada, J., Henríquez, G., Barriga, O., Hernández, L., Romero, J., Bracho, N., Raymond, E., Cárcamo, H., Gavarotto, C., Arnold, M., Pájaro-Huertas, D., Martínez, A., Ríos, F., Scríbano, A., y Toledo, U. (2007). Epistemología de las ciencias sociales: breve manual. Recuperado de:_https:/ /doi.org/10.34720/ n8xe-st04.

Sánchez, A. (1993). La ideología de la neutralidad ideológica en las ciencias sociales. España: Zona Abierta.

Torres, A., y Carrasco, J. (2008). Al filo de la identidad. La migración indígena en América Latina. Ecuador: Crearimagen.

Vergara del Solar, J., Vergara Estévez, J., y Gundermann, H. (2010). Elementos para una teoría crítica de las identidades culturales en América Latina. Utopía y Praxis Latinoamericana, 15(51), 57-79. Recuperado de: https:/ / www.redalyc.org/pdf/279/27916299005.pdf.

Verneaux, R. (1997). Epistemología general o crítica del conocimiento. Curso de filosofía tomista. Barcelona: Herder.

Zizek, S. (2003). El sublime objeto de la ideología. Argentina: Siglo XXI Editores.

Cómo citar este artículo:

Hernández Vélez, A. (2020). La identidad epistemológica de los estudiantes de doctorado en Educación. IE Revista de Investigación Educativa de la REDIECH, 11, e816. doi: http://dx.doi.org/10.33010/ie_rie_rediech.v11i0.816. 